\title{
Women Experience Respect and Disrespect during Childbirth in Tertiary Teaching Hospital. Southwest Part of Ethiopia: Participant Observation, qualitative study
}

\author{
Hirut Megersa \\ St. Paul's Hospital Millennium Medical College \\ Lisbeth Thoresen \\ University of Oslo \\ Belayneh Lulseged \\ St. Paul's Hospital Millennium Medical College \\ Anne Karin Lindahl ( $\sim$ anne.karin.lindahl@ahus.no) \\ University of Oslo
}

\section{Research Article}

Keywords: developing countries, disrespect and abuse, facility-based childbirth, Ethiopia, referral hospital

Posted Date: January 6th, 2021

DOI: https://doi.org/10.21203/rs.3.rs-138289/v1

License: 두 (i) This work is licensed under a Creative Commons Attribution 4.0 International License. Read Full License 


\section{Abstract}

Background: Disrespect and abuse during facility-based childbirth is a negative experience for women in developed and developing regions. Types and drivers of disrespect and abuse vary based on study settings. Few studies have explored disrespect and abuse in tertiary teaching hospitals settings. Therefore, the aim of this study was to explore women experience of disrespect and abuse in one of tertiary teaching hospitals in southwest part of Ethiopia.

Method: Qualitative participant observation was used as a data collection method. Fieldwork was conducted in the labour ward, the normal postnatal ward, and the maternity recovery room for three consecutive months in Jimma University specialized teaching hospital. The participant observation units were selected purposively, and 53 episodes of women in labour and 33 episodes of postpartum women were observed. The data were organized and analysed using NVIVO qualitative analysis software.and we used the thematic analysis method.

Results: Three categories of themes were identified: disrespect and abuse, contributor themes, and respectful themes. Disrespect and abuse themes were physical abuse, poor communication, non-consented care, lack of privacy, lack of confidentiality , neglected care, loss of autonomy and lack of companion,. Contributor themes for disrespect and abuse were provider-related, health system-related, and women-related themes. Provider-related themes were lack of respect among providers and lack of collaboration and communication. Health-system related themes were poor human resource management, scarcity of equipment and supplies, and wastage of supplies. A women-related contributor was women passivity. Respectful care themes were in two categories: respectful provider and respectful facility cultures. Respectful provider practice includes: timely evaluation at admission, being with women, supportive care, and teamwork during emergency. Respectful facility culture included postpartum companion and free delivery service.

Conclusion: The findings of this study indicate that women experienced disrespect and abuse at a teaching health care facility. Policy makers, administrators, and quality improvement initiative activities need to address the identified contributors to improve women's experience in health care facilities.

\section{Background}

Pregnancy and childbirth-related complications are contributors to maternal deaths and disabilities worldwide. On average, there are 12 times more maternal deaths in developing countries than in developed countries[1]. In sub-Saharan Africa, the average maternal mortality rate (MMR) is even higher: 546 maternal deaths per 100,000 live births [1]. In Ethiopia, recent maternal mortality accounts for 401 maternal deaths per 100,000 live births[2].

Facility-based childbirth is recommended as a key strategy for reducing maternal mortality. However, it has been shown to be difficult to achieve fully in developing countries. Many factors may contribute to this difficulty, such as the experience of disrespect and abuse (D\&A) [3-6]. D\&A is also considered a human rights issue and categorized as a violation of women's right to life, health, and quality care $[7,8]$.

D\&A during childbirth may be categorized into seven types: physical abuse, non-consented care, non-confidential care, non-dignified care, discrimination, abandonment of care, and detention in healthcare facilities[3]. Characteristics of D\&A are complex in nature and reflect the relationships between the health system, the women, and the individual health care providers $[4,8]$ D\&A may not only occur at the individual health care provider level, but also occur due to poor performance of the health system[3, 4, 9]. For example, at the health system level, inadequate facility infrastructure, poor supervision and management may result in women's experience of D\&A. From interpersonal factors of individual provider behaviour resulting in disrespectful care [3, 4]. Experience of D\&A during childbirth affects women's health as well as their health-seeking behaviours. There is evidence that such experiences have a negative impact on women's preference for facility-based childbirth for subsequent pregnancy for the women and friends in their community $[3,4,10]$. Because women share these poor experiences with their friends, it leads to poor community perceptions of care in the facility, which deters other women $[4,5,10]$.

The Ethiopian government implemented several interventions to increase the utilization of facilities for childbirth. In Ethiopia, geographical barriers that previously hindered access to maternal health services have been improved and the number of health care facilities increased; in all public health facilities, women are exempted from paying for maternal health services[11, 12]. However, utilization of facilities for childbirth in Ethiopia has not significantly improved. For instance, although the government targets a $90 \%$

Page 2/20 
institutional delivery rate [12].The current institutional delivery rate in Ethiopia is $48 \%[13]$. There are large differences between urban and rural regions for example, $70 \%$ of urban births take place in a health facility, only $40 \%$ in rural areas [13].

These findings indicate that improvement of access to facilities for women is not sufficient for improving utilization. Therefore, improving women's experience of care during labour and delivery is a key strategy for consistent utilization of facility and subsequent reduction maternal mortality[11]. Studies on D\&A are important to identify the gap and to recommend interventions based on the findings. However, previous reports on D\&A have mostly focused on subjective experience of D\&A[14-17] and studies reporting observed incidents are limited.

Quantitative studies used structured observation in other countries as well as in Ethiopia [18-20]. Also, one qualitative study in Tanzania explored D\&A using the participant observation method [21]. Most previous studies on D\&A in Ethiopia focused on quantitative measurements of reported $D \& A[14,15,18,19,22,23]$.

Findings based on subjective experience have their own limitations in identifying hidden and unreported contributors to D\&A. Furthermore, findings on D\&A from teaching and referral health care facility have multiple benefits. Moreover, tertiary hospitals serve as teaching hospitals for medical students, specialist doctors, and midwives and referral hospital for women. Identifying the form of D\&A and its contributors is essential to addressing and recommending possible interventions that improve the experience of care and clinical teaching environment.

Therefore, the aim of this qualitative participant observation of women during labour, delivery, and immediate post-partum in one teaching hospital in the southwest part of Ethiopia was to identify the research gap in D\&A and its contributors in referral and teaching hospitals and to recommend possible interventions based on findings that may be applicable in similar settings.

\section{Methods}

This study is part of a PhD research project on D\&A during childbirth, consisting of empirical data from observations of childbirths, individual interviews and focus group interviews. Here, we describe the method part the first phase which is participant observations.

\section{Study setting}

The study was conducted at a Jimma University specialized teaching hospital. It is a tertiary hospital located in Jimma zone about $352 \mathrm{~km}$ southwest of Addis Ababa, Ethiopia. This hospital was selected purposively because it provides services for both urban and rural populations referred from primary and district health care units from diverse geographical locations in the southwest part of Ethiopia and for being a teaching hospital. The hospital has an average of 750 deliveries per month. Midwives, residents, nurses and senior obstetricians were working in this unit at the time of the study. Observations were conducted in labour and delivery wards, normal postnatal wards and in a maternity recovery room of the study hospital. Study was conducted from October 2017 to the end of January 2018.

\section{Study design}

Qualitative participant observation was conducted to explore D\&A and its contributors in a naturalistic environment. Participant observation provides us with rich data on how women giving birth are actually treated, how procedures and communication are handled, and how the environment contributes to D\&A of the women.

\section{Sample size and sampling technique}

Women who admitted in the labour ward, post-natal ward and maternity recovery room of the study hospital were purposively selected to be involved in participant observation. A total of 53 women in labour and 33 postnatal women with were participated until data saturation. Individual health care providers were not selected as a participant. Because in the study hospital multiple health care provider care for a woman at a time. Sometimes a provider assigned to provide care for multiple women. Provider's actions for a woman documented as an aggregated information on the field note. 


\section{Data collection}

First author (HM) conducted participant observation. After health care providers and department heads had been informed about the research the participant observer was entered in to the labour ward. Participant observation took place in three locations: first and second-stage labour rooms, normal postnatal rooms, and maternity recovery rooms. The observations were conducted on women who were in labour from admission until the end of delivery, for women after spontaneous deliver observed from the first hour after delivery to discharge, and for women who underwent caesarean section, the observations were conducted when they were in $\mathrm{n}$ the recovery room until they transferred to maternity ward. The first author (HM) explained her role as a researcher to the women, and she only remained in the room when the women had given their consent and she observed a consented woman at a time. Observations were conducted in three shifts interchangeably during morning, afternoon, night, and weekend shifts. All observations were documented as short field notes and extended with more detailed information later on the same day. The women in labour were observed for 266 hours average 5 hours with a minimum 1-hour and maximum 11 hours. The post-partum women experience observed for 59 hours. A single episode of observation on a woman includes, all procedures that performed for a woman, woman and health care provider's conversations and provider with provider conversations on a woman care. Also observation includes physical condition of facilities, staffing, supplies and equipment in relation to women care. The researcher (HM) put herself as "The observer as participant stance enables the researcher to participate in the group activities as desired, yet the main role of the researcher in this stance is to collect data, and the group being studied is aware of the researcher's observation activities"[24]. In such role the researcher (HM) was mainly involved in other activities in emergency situations to help provider on equipment and supplies while they were doing lifesaving tasks.

\section{Data analysis}

We use Braun and Clarke's qualitative thematic analysis approach[25]. First, all field notes from participant observations were coded line by line by the first and third authors. Before the final codebook was developed, the initial code list was discussed by the two authors (HM: first author, BL: third author). The remaining field notes were then coded based on the developed codebook. To handle the data in a more manageable manner, NVivo 11 qualitative data analysis software used for merging and categorizing codes. Final themes were interpreted based on Bowser Hill categorises of D\&A, white ribbon respectful maternity care charter and Bohren systematic review typology (table 2 in additional file 1).

\section{Ethical considerations}

Ethical approval for this study was obtained from the Regional Committee for Medical and Health Research Ethics of South East Norway, section B (ref 2017/1050b) and St. Paul's Hospital Millennium Medical College institutional review board reference number (PM23179/25/9/2017) Addis Ababa Ethiopia. . All participants were informed of the voluntary nature of participation and their right to withdraw at any stage of observation. The study objective and method were described to the participants, and their informed consent was obtained before the observation. Confidentiality was assured by assigning each participant a number during data analysis.

\section{Results}

\section{Socio demographic characteristic of participants}

From a total of 86 women their experience observed during labour and after delivery; the majority 46 were from rural/ semi urban areas, most women were 28 were in the age group 25-29, regarding their educational status most 25 women had secondary education, 19 were illiterate and 10 had university degree (Table 1). 
Table 1

Distribution of participants based on Sociodemographic characteristics.

\begin{tabular}{|c|c|c|c|}
\hline \multirow[t]{3}{*}{ Characteristics } & \multicolumn{2}{|l|}{ Participants } & \multirow[t]{2}{*}{ Total } \\
\hline & Labour & Postpartum & \\
\hline & Number $=53$ & Number = 33 & Number $=86$ \\
\hline \multicolumn{4}{|l|}{ Address } \\
\hline Urban & 27 & 13 & 40 \\
\hline Rural/Semiurban & 26 & 20 & 46 \\
\hline \multicolumn{4}{|l|}{ Age } \\
\hline $18-19$ & 5 & 7 & 12 \\
\hline $20-24$ & 13 & 10 & 13 \\
\hline $25-29$ & 20 & 8 & 28 \\
\hline 30 and above & 15 & 8 & 23 \\
\hline \multicolumn{4}{|l|}{ Educational status } \\
\hline Illiterates & 10 & 9 & 19 \\
\hline Primary & 12 & 5 & 17 \\
\hline Junior secondary & 9 & 6 & 15 \\
\hline Secondary & 16 & 9 & 25 \\
\hline University Diploma degree & 6 & 4 & 10 \\
\hline \multicolumn{4}{|l|}{ Para } \\
\hline Primipara & 29 & 18 & 47 \\
\hline Multipara & 21 & 10 & 31 \\
\hline Grand multipara & 3 & 5 & 8 \\
\hline
\end{tabular}

\section{Themes identified from participate observation field note}

Three categories of themes were identified from the analysis of participant observation field note; D\&A themes, contributors themes and respectful themes. (Figure 1)

\section{Disrespect and abusive experience of women}

Themes identified as D\&A included physical abuse, poor communication, non-consented care, lack of privacy, lack of confidentiality , loss of autonomy, neglected care, and lack of companion.(Figure 1).

\section{Physical abuse}

Episiotomy without local anaesthesia was identified as D\&A themes under the category of physical abuse. During their admission for labour and childbirth, the women experienced painful procedure that this study categorized as physical abuse. Some procedures, such as episiotomy, were performed without local anaesthesia while the women felt and verbalized pain. The following field note indicates such an occasion.

A provider was monitoring a woman in labour, when she suddenly pushed down and the baby came at the external genitalia.... Then, the provider put on a pair of surgical gloves, took scissors from the pack of equipment, and made an episiotomy without local 


\section{Poor communication}

Poor communication included interactions that undermine the woman's preference or need, such as women's urge or need to change their position, to know discharge time, and their request for beddings and birth companions. In some observed women's requests, the provider did not respond to the women in an acceptable manner.

A woman asked a midwife, "Please give me beddings?" The midwife laughed at the woman and she said, "No, we don't have beddings". (Field note, post-natal observation (PNO- 06)

Rather than explaining the reason for not allowing a companion, the provider responded to the women with inappropriate words that undermine the woman's concern.

A woman asked a health care provider, "Please allow one attendant to assist me on baby care-l cannot take care of my baby because my hands are dirty and this intravenous fluid lines restricts my movement". The provider replied, "Do not talk too much. I told you before, we cannot allow attendant for the women in this unit". (Field note, PNO -11)

\section{Non-consented care}

Clinical procedures and physical examinations were performed on women without providing information to the women or asking their permission. For instance, women were not informed about the purpose, risk, and benefits of procedures such as physical examination medication, lab investigation and caesarean section. Usually, before procedures and examinations, the provider only requests the women to comply for intended procedures. As the following field note indicates, procedures were performed abruptly without notifying the women.

During the admission of a woman to the first stage room, the health care provider monitoring her labour said to her "Open your legs and uncover your lower body part". The woman opened her legs and undressed her lower body part. He took one surgical glove from the bedside table, put it on one of his hands, and performed a vaginal examination. ((Field note, OBL-28)

Sometimes, providers performed procedures on women during labour without notifying them. Women recognized it when they felt the pain, as the following field note indicates.

A provider assisting in a woman's delivery consistently encouraged her to push with each contraction. Suddenly, he took a scissor from the equipment set and performed an acute episiotomy incision on the woman's perineum. She cried and moved away from him.

Some women were requested to sign written consent forms without sufficient information for caesarean sections. For instance, a provider informed a woman of his decision and immediately insisted she signed a written consent without asking for her agreement.

After performing a physical examination of a woman.....Subsequently, he said to her, "To save the life of your baby, we need to perform a surgical procedure". Then, he attached the informed consent form to her clinical record. Addressing her, he said, "Put your signature here," and she signed the consent form. (Field note. OBL-16)

\section{Lack of privacy}

Women's physical privacy was not protected during labour and delivery. For instance, in the labour ward, procedures, and examinations were performed on women in an open space. Also, women were repeatedly uncovered during physical examination in the view of others. In the case of unpredicted second stage of labour, women's delivery was assisted without a privacy

In a shared room, a provider put on surgical gloves and said to a woman, "Lay down on your back and bend your leg". The woman did as instructed, and the provider performed vaginal examination in the presence of other women and health care providers. (Field note OBL-01). 


\section{Lack of confidentiality}

Providers unintentionally violated women's confidentiality during labour and delivery. The health care providers discussed women's health status, including HIV status, in a shared ward with their co-workers, as the following field note indicates.

One of the health care providers said to his co-workers, "She is an RVI case; take care of yourself", while referring to a woman admitted to the labour ward. After 45 minutes, a midwife came from another room and asked the providers, "Which one of these women is reactive?" One of the residents showed the midwife the bed to which the woman had been admitted. (Field note. OBL-30)

\section{Loss of autonomy}

Women were not involved in decision-making for procedures during labour and delivery. For instance, they did not request a provider's explanation about procedures. However, they responded to all instructions when providers asked them to adopt a position for subsequent procedures. Sometimes, the women's concerns about mobility were ignored.

After a woman was transferred to the delivery bed, a provider awaiting the birth of her baby for one hour said to his co-worker, "I need forceps to assist her", referring to the woman. Then, said to her, "I am going to assist you". The woman did not respond to him before he subsequently applied his forceps and assisted her delivery. (Field note. OBL-29)

\section{Neglected care}

Women's need for supportive care during labour and delivery was neglected. Specifically, women's comfort, hygiene, post-partum care, and postoperative care were neglected. The following field observation note of recovery rooms indicates the neglect of women's intravenous fluid lines after caesarean sections. For instance, intravenous fluid tubes were left open while connected to empty fluid bags.

A woman's attendant in a recovery room said to a health care provider, "Please change this intravenous fluid bag", pointing to an empty fluid bag. However, the provider left the room without responding to the attendant's concern. (Field note, recovery room observation (RRO) 01)

Further, post-operative clinical care of the women was neglected, including laboratory investigations that needed immediate clinical intervention.

A woman's attendant said to a health care provider, "My daughter is Rhesus negative. She has medications to take after she receives the result of her baby's laboratory test results. However, a blood sample was not taken from her baby." The provider responded, "Inform another health care provider", and left the room. (Field note, RRO -02)

Women's comfort after delivery was neglected for clinical monitoring. For example, some women stayed on the delivery bed for more than two hours for observation.

A postnatal mother said to a health care provider, "Please transfer me to another room". Again, she said, "You see I cannot extend my leg on this bed and did not sleep throughout last night because of the cry of other women in labour". The provider did not respond to her concerns. (Feld note, PNO -01)

\section{Lack of companion}

Birth companions were not allowed in the labour and delivery room. For instance, women's attendants were told to stay outside the labour room until the women were transferred to postnatal rooms.

When a woman reached the labour room, a provider told her to lie down on her back, pointing to a bed in the labour room. He then told the woman's companion to stay outside, and immediately, the companion left the room. (Field note, OBL 47). 


\section{Contributors to D\&A}

Contributors to D\&A during childbirth were classified into three main categories: provider-related factors, health facility-related factors, and women-related factors. (Additional file 1)

\section{Provider-related contributor}

Provider-related contributors include lack of respect and lack of collaboration among health care providers working on the same women.

\section{Lack of respect among providers}

Health care providers did not respect each other while they were in the same unit and working for the same women. For instance, residents did not use midwife assessment findings for women care. Sometimes, while the midwife monitored labour, residents interrupted them without asking for an excuse to interrupt. Similarly, sometimes the midwife ignored the resident's verbal orders.

While a midwife was monitoring a woman in labour, a medical resident came in and told the woman to lie down in her back. The woman laid down in her back and the midwife left the place for the resident. Subsequently, the resident performed a physical examination of the woman. (Field Note, OBL-03)

\section{Lack of communication and collaboration}

There were no formal communications between the midwives and the residents about the women's clinical condition. For instance, a midwife was not involved in clinical communications about diagnosis, obstetric decisions and case handover. Such practice of providers contributing to D\&A included unnecessary examinations and neglected care.

The resident performed an examination of a woman who cried due to labour pain in a shared labour ward and moved to another woman. After 10 minutes of his examination, a midwife came to the same woman. She performed the same examination and left the room. (Field note OBL 10).

\section{Health system-related contributors}

Health system-related contributor themes included poor human resource management, scarcity of equipment and supplies, and wastage of supplies.

\section{Poor human resource management}

Subthemes included a lack of recognition of professional skills, overburdened responsibility, lack of supportive supervision and inconsistent supervision of trainees.

\section{Lack of recognition of professional skills}

Lack of recognition of professional competencies of some health care providers contributed to D\&A. Health care professionals from different disciplines were assigned to the same labour ward; however, some of them worked below their professional scope of practice. For instance, midwifes were not regularly involved in monitoring labour, conducting delivery, or in shift handovers. They were more engaged in equipment preparation and discharge procedures, even during high case load time. This culture of the facility caused midwives to ignore the women's obstetric care needs and focus on other tasks, as in the following field note:

While a medical student was monitoring a woman in labour, spontaneously, the woman pushed down and delivered on her bed. He did not anticipate the imminent birth and was not prepared for it. At the same time, four midwives were preparing gauze in a room that

Page $8 / 20$ 
Some of the midwives focused more on equipment preparation than clinical care. For instance, a midwife could postpone medication time to prepare delivery equipment. This included medications for preventing convulsion.

A resident asked a midwife to administer $\mathrm{MgSO}_{4}$ to a mother; the midwife replied affirmative, although she continued with her equipment preparation. After 40 minutes of the verbal order, another resident asked if $\mathrm{MgSO}_{4}$ had been given to the mother. "Who gave her the medication? By whom and when?" The first resident replied, "Sr. X has given to her", referring to the midwife. But the medication had not been given to the woman. The midwife returned after she was finished with the equipment preparation to administer the MgSO4 to the mother. (Field note, PNO-21)

\section{Overloaded responsibility}

Some health care providers took part in most of the tasks carried out in the labour room. For instance, the residents evaluated women at admission, monitored, and conducted all types of delivery. Thus, the work burden on them contribute to non-consented care, neglected care and rushed procedures.

Four medical residents were assigned to a labour room. Two of them worked in the operation theatre on a woman with obstetric complications. One of the residents assisted another woman in delivery. The last resident alone monitored five women admitted to the first stage room. (Field note, OBL-O6)

\section{Inconsistent supervision of trainees}

Trainee assignments with inadequate supervision contribute to D\&A. When residents were occupied with other emergency tasks, students monitored women in labour with full responsibility and subsequently failed to detect imminent birth. Therefore, they performed episiotomy without local anaesthesia and conducted delivery without physical privacy.

While a medical student monitored a woman's labour, she pushed downward spontaneously and the baby's head became visible. However, the student did not anticipate imminent delivery and was not prepared for it. Afterward, he put on surgical glove, performed episiotomy without local anaesthesia, and assisted the delivery without privacy curtains. (Field note, OBL-02)

\section{Lack of supportive supervision}

Lack of supervision contributed to D\&A. Some health care providers were not present at their assigned workplace, and available providers were overstretched to cover an entire unit's tasks. As a result of their high workload, the available health care providers ignored some of the women.

A resident was assigned to monitor women labour admitted to two labour rooms. He came to a woman in one of the labour rooms and checked foetal heart rate. At the same time, a test tube was placed on her bed for a routine blood tests; however, he could not take the sample from the woman. (Field note, OBL-17)

Some health care providers intentionally reported wrong diagnoses to postpone their responsibility for the next shift provider. Such practice of providers resulted in unexpected delivery without privacy and dignity in which women's bodies were exposed to other women admitted to the same room and health care providers.

During a shift handover, a woman's labour had been reported as latent first stage of labour. However, after 15 minutes of the handover, she delivered her baby on her bed in a shared room in the presence of other women (Field note, OBL-24).

\section{Scarcity of equipment}

Scarcity of equipment resulted in D\&A in the form of abandonment of timely care, lack of hygienic care, and unnecessary procedures. For instance, a shortage of sterile surgical equipment and operating theatre tables frequently delayed emergency caesarean sections 
for 15-30 minutes. Scarcity of vital sign equipment affected regular monitoring after delivery. Sometimes woman insisted on leaving her own bed for another woman in advanced labour after her admission. When she refused, the providers talked to her rudely, insisting she complied. In the following field note, shortage of maternity operation theatre table and scarcity of sterile equipment delayed emergency caesarean section and resulted in a fatal consequence

A resident identified that the cord was blocking a second twin after he assisted a woman's first twin. He immediately instructed the woman to adopt the knee-chest position. His co-worker informed the operation theatre team about an emergency case that required an urgent caesarean section. However, the maternity operation theatre table was occupied by another woman. To expedite access, the health care providers informed the major operation theatre unit responsible person of the mother's situation, who, unfortunately, responded that sterile equipment was not ready. (Field note-OBL 31)

Scarcity of equipment used in assisting delivery, such as obstetric forceps and vacuum extractors, resulted in unnecessary caesarean section. Sometimes the essential equipment was not ready for use, and providers became aware of the unavailability of the particular equipment when he/she wanted to use it, as demonstrated in the following field note.

A resident had decided to assist a woman labour with obstetric forceps and asked the mid wife to retrieve them. She responded that the forceps were sent to another unit for sterilization. The midwife left the room to bring the forceps from the autoclave that was located in the major operation theatre. She came back after a few minutes and told the resident the equipment was not ready for use. He then requested a vacuum extractor in place of the forceps. The midwife brought him a vacuum extractor, which, when subsequently applied, was not functional. Finally, he decided to assist the delivery with a caesarean section. (Field note, OBL 48)

\section{Scarcity of supplies}

Scarcity of supplies caused women to stay in unhygienic and non-dignified conditions of labour rooms. Most women in the labour ward stayed without beddings, sometimes undressed, or with their soiled clothes, and in some rare circumstances, admitted to the bare floor. The majority of the women were admitted without bedding in the labour and postnatal wards, as the following field note indicates.

A health care provider instructed a woman to lie down in her back on some bed. The bed was without beddings, and its mattress was covered with a plastic sheet. (Field note, OBL-01)

\section{Wastage of supplies}

Wastage of supplies resulted in shortage and subsequently contributed to D\&A. For instance, new bed sheets were cut into small pieces to pack equipment, and after a single use, they were used to wipe blood from the floor. Stained and difficult to reuse, such patterns created wastage of bedding in the labour wards. The following field note indicates this practice.

A cleaner placed some gowns and bed sheets to cover some blood splashed on the floor, and she stood on it. Subsequently, she wiped blood from the bed with pieces of the bed sheet. (Field note, OBL-14)

\section{Women-related contributors}

\section{Passivity}

Women were passive participants in the care they received during labour and delivery. Most of the women did not ask providers for information about examinations and procedures. The providers simply instructed them to adopt certain positions for each subsequent procedure without informing them.

A health care provider told a woman to uncover her abdomen and flex her leg. The woman uncovered her abdomen as she was instructed. He examined her abdomen and listened for foetal heart rate with a fetoscope. Then, he put on surgical gloves and asked the woman to open her legs, which she did as he proceeded to a vaginal examination. (Field note, OBL 02). 


\section{Respectful care}

Some provider practice and facility culture were considered as respectful care, such as being with the women during labour, timely clinical care at admission, supportive care, and provider collaboration in obstetric emergency. Respectful facility culture includes birth companion after deliver and free maternity service.

\section{Being with the women}

At least one health care provider-a medical intern or resident-are often assigned to a woman in labour. In most cases, at least one trainee monitored the women throughout their labour. Thus, women were not left alone during their labour and delivery.

He [health care provider] sat on a chair close to a labouring woman's bed, placed his hand on her abdomen and counted uterine contractions. He repeated the same examination at regular intervals. (Field note. OBL-03)

\section{Timely clinical care}

Women receive timely clinical care throughout their stay in the labour ward. In majority of women provider evaluate their clinical condition throughout their labour and provide clinical care according to women need without delay unless constraints beyond provider was present during women admission.

\section{Supportive care}

Some women received supportive care from the health care providers after delivery. For instance, some health care providers brought clothes, food, and fluid from the women's attendants at the reception and accompanied the women during transfer from the labour room to the postnatal room.

When a day shift midwife came close to the postnatal mother, the mother requested, "Please bring me clothes from my relatives waiting at the reception. The provider went to the labour ward entrance, collected some clothes from the woman's attendant, and brought them to her. (Field note. PNO-14)

\section{Teamwork in emergency}

In emergency situations, women receive collaborative care from health care providers. Such practice was common in emergency cases such as uterine rupture. Residents collaborated with midwives and nurses to save the lives of the women.

The physician who was assigned to the room immediately transferred the woman from a stretcher to a bed. One health care provider took the obstetric history of the woman and another measured vital signs. Subsequently, other health care providers opened two intravenous lines through her arms and initiated the fluid. (Field note. OBL-26)

\section{Respectful facility culture}

Respectful facility culture includes birth companion after deliver and free maternity service.

\section{Postnatal companion}

For women admitted for delivery, a birth companion was allowed after they were transferred to the postnatal ward.

After a woman changed her soiled clothes, the midwife transferred her to the normal postnatal room and called for one birth companion from the woman's relatives to stay and assist her. (Field note. PNO - 10). 


\section{Free maternity service}

Women did not have to pay for maternal health services such as spontaneous delivery, caesarean section, instrumental delivery, or medications. Therefore, women were not detained for lack of payments. Usually, at discharge, they were given a letter of clearance that allowed them to leave the facility without receipt showing payment.

A midwife provided a letter to all postnatal mothers at discharge that authorised them to go home without payment for the received service.

\section{Discussion}

We conducted qualitative participant observation to explore D\&A during childbirth in one of the referral and teaching hospitals in the southwest part of Ethiopia. We observed the experience of women in labour and after delivery. Our observation included a total of 86 women's experiences until data saturation: 53 women's experiences were observed during labour and 33 women's experiences were observed after delivery. We used a thematic analysis method [25] and identified three main categories of themes: D\&A, contributors to $D \& A$, and respectful care theme. D\&A themes included physical abuse, poor communication, non-consented care, lack of privacy, lack of confidentiality, loss of autonomy, neglected care, and lack of a birth companion.

Regarding specific forms of D\&A, our study indicated that during childbirth, women experienced physical abuse. For example, some providers performed perineal incisions on women during delivery without local anaesthesia. We considered this women's experiences as violations of women's rights to be free from harm and ill-treatments categorized as physical abuse [8, 26]. [3, 20, 22]. However, this forms of physical abuse were unintentional because the provider did not perform them to obtain the women's compliance. Prior studies reported providers slapping, pinching, or restraining women for compliance [22, 27-30] .

Another form of D\&A identified in our study was neglected care. Women were neglected after delivery in postnatal wards and recovery rooms. Previous studies showed that women were often neglected during labour and delivery [21, 31-34]. This difference might be due to differences in study settings and observation methods used. The teaching hospital setting of our study had trainees who were interested in monitoring labour and conducting delivery. Therefore, in such an environment, women's neglect during labour might not be a common experience.

In the present study, we used participant observation as a data collection method; however, most prior studies used the interview method as the basis for reporting women's experiences. Women are naturally perceived to be more vulnerable during labour than after delivery, and they might be more sensitive to experiences of neglect during labour they report such experience as D\&A. However, neglect after delivery might not be as frequently reported as D\&A on interviews because of the joy being celebrated. The neglect we observed after normal delivery and caesarean sections were not undermined since most complications that end in maternal deaths occur after delivery [35-37].

We found poor communication between women and providers during labour and delivery as one form of D\&A practice. Such women's experiences were reported in Bohren systematic review as a mistreatment in the form of poor rapport [4] and prior studies reported a as D\&A [31, 38-42]. Further, effective health care provider-patient communication fosters a positive relationship and is essential to providing quality maternal health service[43].

The lack informed consent process was another form of D\&A in our study. For instance, women did not consented for routine procedures and they signed written consent forms before they received sufficient information on the risks of caesarean section. This is consistent with the findings of Bohren systematic review and other study findings $[3,4,9,14,15,21,31]$. Informed consent maintains women's rights to autonomy and protects the patient from undesirable procedures [44]. Further, studies are needed to understand the rationales for this practice from the provider's perspective, which is an ongoing part of this research project.

Women's privacy was not maintained during labour and delivery. In our study, the presence of a large number of trainees at a time, poor room design, and scarcity of curtains contributed to lack of privacy during first stage of labour, as previously reported studies [4, 21, $27-29,45,46]$. [47]. However, privacy and confidentiality are the main parts of maternity care [48, 49]. [50, 51].Lack of privacy during labour has several implications and is a reason for women's dissatisfaction avoidance of facility-based child [52, 53]. 
We categorised the contributors to D\&A into three main themes: provider-related contributors, health system-related contributors, and women-related contributors, similar to other previous findings, including the report of a systematic review and landscape analysis $[3,4$, 28]. Under the poor human resource management of health system-related contributors, we identified subthemes such as lack recognition for professional skill, multiple responsibility for residents, lack of supportive supervision to the staff, and inconsistent follow-up of trainees. These findings agree with prior findings [21, 22].

In the present study, lack of respect among providers was identified as a provider-related contributor to D\&A. We found that midwives acted as passive participants in women's care, similar to the report of a previous study [57]. In contrast, the residents were overburdened with routine care and struggled to cover all the unit tasks. Such health care providers' practices contributed to D\&A.

Furthermore, lack of recognition for the professional skills for midwives contributed to D\&A. For instance the midwives in the labour wards were not assigned to monitor labour and to assist delivery. During high caseload circumstances, the residents informally delegated their responsibility to medical students with inadequate supervision. Subsequently, medical students performed procedures that were considered D\&A. In contrast to previous studies that reported a shortage of human resources as a contributor to D\&A [17, 24, $27,31,58]$.

Our study found that lack of inter-professional collaboration was a contributor to D\&A. The lack of collaboration between midwives and residents created a disorganized working environment, in which some health care providers were overburdened while others stayed idle. Similarly, prior reports identified lack of collaboration as a challenge for unsuccessful health services [59] Therefore, our findings show that effective human resource management may contribute to reducing D\&A. Moreover, modern health systems require collaboration to provide quality care [60]. Specifically, in maternal health services, collaboration is essential for safety. However, poor collaboration are the known causes of poor outcomes [61].

We also found that scarcity of equipment and supplies was another contributor to D\&A. Occasionally, unnecessary procedures were performed on the women due to scarcity of equipment. Emergency caesarean sections were sometimes delayed after the decision and women stayed without beddings. These findings agree with prior reports $[4,15,17,46,64]$ However, in our study, it did not result in detention and verbal abuse as prior findings [16]

In present study, women's passivity contributed to D\&A. Women did not ask for information about procedures from the health care providers; rather, they complied with multiple providers' commands for procedures. This provider's practice and women's behaviour contributed to non-consented care. A prior study reported women's lack of knowledge about their rights and lack of legal redress mechanisms as contributors to women's passivity [3,33].

\section{Conclusion}

This study is one of the few qualitative studies that have explored women's experience of D\&A during intrapartum and early postpartum periods in teaching hospital in the southwest part of Ethiopia. Our findings add to the existing body of evidence on D\&A during labour, delivery, and immediate post-partum within teaching hospital. Most of our findings on drivers of D\&A are consistent with the findings from previous studies. However, poor human resource management and lack of provider collaboration were unique findings of the present study. Furthermore, to the best of our knowledge, this is the first study to specifically investigate women's experiences of D\&A using participant observation in the labour ward of a tertiary teaching hospital. Our findings will serve as input for the Ethiopian government's efforts and other developing countries to improve maternal health services. Furthermore, these results will help the efforts of maternal health stakeholders intended to improve women's experience in teaching hospitals.

\section{Recommendations}

To reduce women's experiences of D\&A in teaching hospital settings, interventions are needed to improve the lack of professional collaboration, poor human resource management, lack of recognition of midwifery skills, and scarcity of equipment and supplies. Providers' training on teamwork and collaboration in health care facilities might improve women's experiences of care. Recognizing midwifery skills and actively involving them in women's care will fill the skilled human resource gap, improve women's experience of care, and reduce the work burden on medical residents in teaching hospitals. Further, women's empowerment of their rights at the community level might be an important intervention area to make the women active participants in their own care. Integrating respectful maternity care concepts as a course on preservice and in-service curriculum is an important strategy. Overall, improving

Page $13 / 20$ 
women's experience of care demands close collaboration among researchers and key maternal health stakeholders. Our findings indicate the need for further research to assess women's opinion and provider perspectives on the identified D\&A and contributors, which is an ongoing study following this paper.

\section{Limitations}

We assumed that social desirability might have altered health care providers' behaviours and the impact on women's experience of care. However, the researcher's several hours of presence in observing disrespectful and abusive experiences in the field reduce such limitations. Purposive sampling might have a possible selection bias. Our study has some strengths. We conducted observations of women's experiences during day, night, and weekend shifts to capture the possibility for differences in the experiences of women during the different shifts. Another strength of our study is that the women's experiences of care were observed from the perspective of an experienced nurse-midwife with more than 20 years' work experience and advanced degrees in maternity and reproductive health nursing. The direct observations provide a rich source of data and first-hand observations of D\&A. The knowledge gained could be transferred to similar settings.

\section{List Of Abbreviations}

D\&A disrespect and abuse, MMR- maternal mortality ratio IRB- institutional review bored, JUSTH -Jimma University specialized Teaching Hospital , UIO- university of Oslo, OBL- observation of labour, PNO- postnatal observation, RRO- recovery room observation .

\section{Declarations}

\section{Ethics approval and consent to participate}

Ethical approval for this study was obtained from the Regional Committee for Medical and Health Research Ethics of South East Norway, section B (ref 2017/1050b) and from St. Paul's Hospital Millennium Medical College Institutional Review Board, Addis Ababa, Ethiopia (with ref. number of PM 23179/25/9/2017). All methods were performed in accordance with the institution's guidelines and regulations. All participants were informed of the voluntary nature of participation and their right to withdraw at any stage of observation. The study objective and method were described to the participants, and a written structured consent form administered in participants' preferred local language (Amharic and Affan Oromo). Their informed consent was obtained before the observation. Confidentiality was assured by assigning each participant a number during data analysis.

\section{Consent for publication}

Not applicable, since there are no details on individuals reported within this manuscript

\section{Availability of data}

The transcripts from which this manuscript was developed are available on reasonable request from the corresponding author

\section{Competing interest}

The authors declare that they have no competing interests.

\section{Funding}

Financial support for this study was provided by NORHED as part of PhD study project from SACCADE Q2A-0A-0484, ETH-13/0024, and UIO project number 200928. The contents of this manuscript are the sole responsibility of the authors and do not necessarily reflect the views of NORHED. The funding body did not have any role in the study design, data collection and analysis, interpretation of data, or in writing the manuscript. 


\section{Authors' contributions}

HM was the lead author involved in the overall conceptual design of the study, carried out the data collection and analysis, prepared the manuscript $B L$ was involved in the data analysis, $A L$ and $L T$ reviewed the conceptual design, supervised the overall process of this research and, reviewed the manuscript. All authors read and approved the final manuscript.

\section{Acknowledgement}

The authors would like to thank all women participated in this study and staffs in the study hospital in Ethiopia. We are very grateful for the support of Dr. Jeanette H.Mangus, Ms. Line Marie Low and other members of Strategic Collaborative Capacity Development in Ethiopia and Africa (SACCADE) program in university of Oslo.

\section{Author's information}

H.M- PhD student at university of OSLO (UIO) institute of health and society and working at Department of nursing, St. Paul's Hospital Millennium Medical College Addis Ababa Ethiopia

A.L- Associate professor, Department of health administration and health economics, Institute of Health and Society, Faculty of Medicine, University of Oslo, Norway and Head of Division of Surgery, Akershus University Hospital, Norway.

L.T- Department of Interdisciplinary Health Sciences, Institute of Health and Society, Faculty of Medicine, University of Oslo, Norway

B.L- Department of public health St. Paul's Hospital Millennium Medical College Addis Ababa Ethiopia

\section{Competing interests}

The authors declare that there are no competing of interests.

\section{References}

1. Organization WH: Trends in maternal mortality: 1990-2015: estimates from WHO, UNICEF, UNFPA, World Bank Group and the United Nations Population Division: World Health Organization; 2015.

2. WHO UJWHO: UNFPA, World Bank Group, and the United Nations Population Division. Trends in Maternal Mortality: 2000 to 2017. Geneva. 2019.

3. Bowser D, Hill K: Exploring evidence for disrespect and abuse in facility-based childbirth: report of a landscape analysis. In: USAIDTRAction Project, Washington, DC. edn.; 2010.

4. Bohren MA, Vogel JP, Hunter EC, Lutsiv O, Makh SK, Souza JP, Aguiar C, Saraiva Coneglian F, Diniz ALA, Tunçalp Ö et al: The Mistreatment of Women during Childbirth in Health Facilities Globally: A Mixed-Methods Systematic Review. PLOS Medicine 2015, 12(6):e1001847.

5. Bradley S, McCourt C, Rayment J, Parmar D: Disrespectful intrapartum care during facility-based delivery in sub-Saharan Africa: A qualitative systematic review and thematic synthesis of women's perceptions and experiences. Social science \& medicine (1982) 2016, 169:157-170.

6. Organization WH: The prevention and elimination of disrespect and abuse during facility-based childbirth: WHO statement. In: The prevention and elimination of disrespect and abuse during facility-based childbirth: WHO statement. edn.; 2014.

7. WHO. Prevention and elimination of disrespect and abuse during childbirth

[https://www.who.int/reproductivehealth/topics/maternal_perinatal/statement-childbirth/en/] 
8. White Ribbon Alliance. Respectful Maternity care the universal rights of child bearing women [https://www.whiteribbonalliance.org/wp-content/uploads/2017/11/Final_RMC_Charter.pdf]

9. Freedman LP, Ramsey K, Abuya T, Bellows B, Ndwiga C, Warren CE, Kujawski S, Moyo W, Kruk ME, Mbaruku G: Defining disrespect and abuse of women in childbirth: a research, policy and rights agenda. Bulletin of the World Health Organization 2014, 92(12):915-917.

10. Bohren MA, Hunter EC, Munthe-Kaas HM, Souza JP, Vogel JP, Gülmezoglu AM: Facilitators and barriers to facility-based delivery in low- and middle-income countries: a qualitative evidence synthesis. Reproductive Health 2014, 11(1):71.

11. Getachew A, Ricca J, Cantor D, Rawlins B, Rosen H, Tekleberhan A, Bartlett L, Gibson H: Quality of Care for Prevention and Management of Common Maternal and Newborn Complications: A Study of Ethiopia's Hospitals. 2011.

12. HSTP.The Federal Democratic Republic of Ethiopia Ministry of Health. Health sector Transformation plan, 2015/162019/20.Federal Democratic Republic of Ethiopia Ministry of health [https://www.globalfinancingfacility.org/ethiopia-healthsector-transformation-plan-201516-201920]

13. Ethiopian Public Health Institute (EPHI) [Ethiopia] and ICF. 2019. Ethiopia Mini Demographic andHealth Survey 2019: Key Indicators. Rockville, Maryland, USA: EPHI and ICF. [https://dhsprogram.com/pubs/pdf/PR120/PR120.pdf]

14. Asefa A, Bekele D: Status of respectful and non-abusive care during facility-based childbirth in a hospital and health centers in Addis Ababa, Ethiopia. Reprod Health 2015, 12:33.

15. Asefa A, Bekele D, Morgan A, Kermode M: Service providers' experiences of disrespectful and abusive behavior towards women during facility based childbirth in Addis Ababa, Ethiopia. Reprod Health 2018, 15(1):4.

16. Abuya T, Warren CE, Miller N, Njuki R, Ndwiga C, Maranga A, Mbehero F, Njeru A, Bellows B: Exploring the prevalence of disrespect and abuse during childbirth in Kenya. PloS one 2015, 10(4):e0123606.

17. Rominski SD, Lori J, Nakua E, Dzomeku V, Moyer CA: When the baby remains there for a long time, it is going to die so you have to hit her small for the baby to come out": justification of disrespectful and abusive care during childbirth among midwifery students in Ghana. Health policy and planning 2017, 32(2):215-224.

18. Rosen HE, Lynam PF, Carr C, Reis V, Ricca J, Bazant ES, Bartlett LA: Direct observation of respectful maternity care in five countries: a cross-sectional study of health facilities in East and Southern Africa. BMC Pregnancy Childbirth 2015, 15:306.

19. Sheferaw ED, Bazant E, Gibson H, Fenta HB, Ayalew F, Belay TB, Worku MM, Kebebu AE, Woldie SA, Kim YM et al: Respectful maternity care in Ethiopian public health facilities. Reprod Health 2017, 14(1):60.

20. Sethi R, Gupta S, Oseni L, Mtimuni A, Rashidi T, Kachale F: The prevalence of disrespect and abuse during facility-based maternity care in Malawi: evidence from direct observations of labor and delivery. Reprod Health 2017, 14(1):111.

21. Shimoda K, Horiuchi S, Leshabari S, Shimpuku Y: Midwives' respect and disrespect of women during facility-based childbirth in urban Tanzania: a qualitative study. Reprod Health 2018, 15(1):8.

22. Banks KP, Karim AM, Ratcliffe HL, Betemariam W, Langer A: Jeopardizing quality at the frontline of healthcare: prevalence and risk factors for disrespect and abuse during facility-based childbirth in Ethiopia. Health policy and planning 2018, 33(3):317-327.

23. Sheferaw ED, Mengesha TZ, Wase SB: Development of a tool to measure women's perception of respectful maternity care in public health facilities. BMC Pregnancy Childbirth 2016, 16:67.

24. Kawulich BB: Participant observation as a data collection method. In: Forum qualitative sozialforschung/forum: Qualitative social research: 2005; 2005.

25. Braun V, Clarke VJQrip: Using thematic analysis in psychology. 2006, 3(2):77-101.

26. Organization WH: WHO recommendations on intrapartum care for a positive childbirth experience: World Health Organization; 2018. 
27. Burrowes S, Holcombe SJ, Jara D, Carter D, Smith K: Midwives' and patients' perspectives on disrespect and abuse during labor and delivery care in Ethiopia: a qualitative study. BMC Pregnancy Childbirth 2017, 17(1):263.

28. Balde MD, Diallo BA, Bangoura A, Sall O, Soumah AM, Vogel JP, Bohren MA: Perceptions and experiences of the mistreatment of women during childbirth in health facilities in Guinea: a qualitative study with women and service providers. Reproductive Health $2017,14(1): 3$.

29. Bohren MA, Vogel JP, Tuncalp O, Fawole B, Titiloye MA, Olutayo AO, Ogunlade M, Oyeniran AA, Osunsan OR, Metiboba L et al: Mistreatment of women during childbirth in Abuja, Nigeria: a qualitative study on perceptions and experiences of women and healthcare providers. Reprod Health 2017, 14(1):9.

30. Moyer CA, Adongo PB, Aborigo RA, Hodgson A, Engmann CM: 'They treat you like you are not a human being': Maltreatment during labour and delivery in rural northern Ghana. Midwifery 2014, 30(2):262-268.

31. Chadwick RJ, Cooper D, Harries J: Narratives of distress about birth in South African public maternity settings: A qualitative study. Midwifery 2014, 30(7):862-868.

32. McMahon SA, George AS, Chebet JJ, Mosha IH, Mpembeni RN, Winch PJ: Experiences of and responses to disrespectful maternity care and abuse during childbirth; a qualitative study with women and men in Morogoro Region, Tanzania. BMC Pregnancy and Childbirth 2014, 14(1):268.

33. Mselle LT, Moland KM, Mvungi A, Evjen-Olsen B, Kohi TW: Why give birth in health facility? Users' and providers' accounts of poor quality of birth care in Tanzania. BMC health services research 2013, 13(1):174.

34. Warren CE, Njue R, Ndwiga C, Abuya T: Manifestations and drivers of mistreatment of women during childbirth in Kenya: implications for measurement and developing interventions. BMC Pregnancy Childbirth 2017, 17(1):102.

35. Say L, Chou D, Gemmill A, Tunçalp Ö, Moller A-B, Daniels J, Gülmezoglu AM, Temmerman M, Alkema L: Global causes of maternal death: a WHO systematic analysis. The Lancet Global Health 2014, 2(6):e323-e333.

36. Organization WH: Strategies towards ending preventable maternal mortality (EPMM). 2015

37. Organization WH: Managing complications in pregnancy and childbirth: a guide for midwives and doctors: World Health Organization; 2017.

38. Ng'anjo Phiri S, Fylkesnes K, Ruano AL, Moland KM: 'Born before arrival': user and provider perspectives on health facility childbirths in Kapiri Mposhi district, Zambia. BMC Pregnancy and Childbirth 2014, 14(1):323.

39. Mehretie Adinew Y, Abera Assefa N: Experience of Facility Based Childbirth in Rural Ethiopia: An Exploratory Study of Women's Perspective. Journal of pregnancy 2017, 2017:7938371.

40. Bhattacharyya S, Issac A, Rajbangshi P, Srivastava A, Avan BI: "Neither we are satisfied nor they"-users and provider's perspective: a qualitative study of maternity care in secondary level public health facilities, Uttar Pradesh, India. BMC health services research $2015,15: 421$.

41. Hartigan L, Cussen L, Meaney S, O’Donoghue K: Patients' perception of privacy and confidentiality in the emergency department of a busy obstetric unit. BMC health services research 2018, 18(1):978.

42. Shakibazadeh E, Namadian M, Bohren MA, Vogel JP, Rashidian A, Nogueira Pileggi V, Madeira S, Leathersich S, Tuncalp, Oladapo OT et al: Respectful care during childbirth in health facilities globally: a qualitative evidence synthesis. BJOG : an international journal of obstetrics and gynaecology 2017.

43. Madula P, Kalembo FW, Yu H, Kaminga AC: Healthcare provider-patient communication: a qualitative study of women's perceptions during childbirth. Reproductive Health 2018, 15(1):135.

44. Hall DE, Prochazka AV, Fink AS: Informed consent for clinical treatment. CMAJ 2012, 184(5):533-540. 
45. Behruzi R, Hatem M, Goulet L, Fraser W: The facilitating factors and barriers encountered in the adoption of a humanized birth care approach in a highly specialized university affiliated hospital. BMC women's health 2011, 11:53.

46. Ishola F, Owolabi O, Filippi V: Disrespect and abuse of women during childbirth in Nigeria: A systematic review. PloS one 2017, 12(3):e0174084.

47. Noroozi M, Zahedi L, Bathaei FS, Salari P: Challenges of Confidentiality in Clinical Settings: Compilation of an Ethical Guideline. Iran J Public Health 2018, 47(6):875-883.

48. Hartigan L, Cussen L, Meaney S, O'Donoghue K: Patients' perception of privacy and confidentiality in the emergency department of a busy obstetric unit. BMC health services research 2018, 18(1):978-978.

49. George J, Bhila T: Security, Confidentiality and Privacy in Health of Healthcare Data. International Journal of Trend in Scientific Research and Development 2019, Volume-3

50. Moskop JC, Marco CA, Larkin GL, Geiderman JM, Derse AR: From Hippocrates to HIPAA: privacy and confidentiality in emergency medicine--Part I: conceptual, moral, and legal foundations. Ann Emerg Med 2005, 45(1):53-59.

51. Beltran-Aroca CM, Girela-Lopez E, Collazo-Chao E, Montero-Pérez-Barquero M, Muñoz-Villanueva MC: Confidentiality breaches in clinical practice: what happens in hospitals? BMC Medical Ethics 2016, 17(1):52.

52. Bitew K, Ayichiluhm M, Yimam K: Maternal Satisfaction on Delivery Service and Its Associated Factors among Mothers Who Gave Birth in Public Health Facilities of Debre Markos Town, Northwest Ethiopia. BioMed research international 2015, 2015:460767

53. Kigenyi O, Tefera GB, Nabiwemba E, Orach CG: Quality of intrapartum care at Mulago national referral hospital, Uganda: clients' perspective. BMC Pregnancy and Childbirth 2013, 13(1):162.

\section{Table}


Table 2

Comparison of identified themes D\&A with prior published D\&A categories and typologies.

\begin{tabular}{|c|c|c|c|}
\hline \multirow{2}{*}{$\begin{array}{l}\text { Present study } \\
\text { findings } \\
\text { Disrespect and } \\
\text { abuse themes }\end{array}$} & \multicolumn{3}{|c|}{ Previously published D\&A categories } \\
\hline & $\begin{array}{l}\text { Bower and Hill } \\
\text { categories of } \\
\text { D\&A[3] }\end{array}$ & Respectful maternity care charter [8] & $\begin{array}{l}\text { Boheren } \\
\text { typology of } \\
\text { mistreatment } \\
\text { [4] }\end{array}$ \\
\hline $\begin{array}{l}\text { 1. Physical } \\
\text { abuse }\end{array}$ & $\begin{array}{l}\text { Physical-abuse } \\
\text { include episiotomy } \\
\text { without anesthesia }\end{array}$ & Freedom from harm and ill treatment & $\begin{array}{l}\text { Refusal to } \\
\text { provide pain } \\
\text { relief and } \\
\text { painful -vaginal } \\
\text { exams under } \\
\text { second order } \\
\text { themes failure } \\
\text { to meet } \\
\text { professional } \\
\text { standard of } \\
\text { care }\end{array}$ \\
\hline $\begin{array}{l}\text { 2. Poor } \\
\text { communication }\end{array}$ & - & Right to information & $\begin{array}{l}\text { Poor } \\
\text { communication } \\
\text { subtheme } \\
\text { under second } \\
\text { order theme } \\
\text { Poor rapport } \\
\text { between } \\
\text { women and } \\
\text { providers }\end{array}$ \\
\hline $\begin{array}{l}\text { 3. Non- } \\
\text { consented care }\end{array}$ & $\begin{array}{l}\text { Non-consented } \\
\text { care }\end{array}$ & $\begin{array}{l}\text { Right for informed consent and refusal, } \\
\text { and respect for choices and preferences }\end{array}$ & $\begin{array}{l}\text { Lack of } \\
\text { informed } \\
\text { consent } \\
\text { process }\end{array}$ \\
\hline $\begin{array}{l}\text { 4. Lack of } \\
\text { privacy }\end{array}$ & $\begin{array}{l}\text { Physical lack of } \\
\text { privacy (Under- } \\
\text { Non-confidential } \\
\text { care) }\end{array}$ & Right to privacy & $\begin{array}{l}\text { Lack of privacy } \\
\text { under health } \\
\text { systems } \\
\text { conditions and } \\
\text { constraints } \\
\text { second order } \\
\text { theme }\end{array}$ \\
\hline $\begin{array}{l}\text { 5. Lack of } \\
\text { confidentiality }\end{array}$ & $\begin{array}{l}\text { Lack of privacy for } \\
\text { woman sensitive } \\
\text { health } \\
\text { information((Under- } \\
\text { Non-confidential } \\
\text { care }\end{array}$ & Right for Confidentiality & $\begin{array}{l}\text { Breaches of } \\
\text { confidentiality: } \\
\text { failure to meet } \\
\text { professional } \\
\text { standard of } \\
\text { care }\end{array}$ \\
\hline $\begin{array}{l}\text { 6. Loss of } \\
\text { autonomy }\end{array}$ & - & Liberty, autonomy, self-determination, and freedom from coercion & $\begin{array}{l}\text { Under third } \\
\text { order theme } \\
\text { poor rapport } \\
\text { between } \\
\text { women and } \\
\text { health care } \\
\text { provider loss } \\
\text { of autonomy } \\
\text { as second } \\
\text { order theme }\end{array}$ \\
\hline $\begin{array}{l}\text { 7. Neglected } \\
\text { care }\end{array}$ & $\begin{array}{l}\text { Abandonment of } \\
\text { Care }\end{array}$ & Right to timely healthcare and to the highest attainable level of health & Neglect \\
\hline $\begin{array}{l}\text { 8. Lack of } \\
\text { companion }\end{array}$ & & Respect companionship during maternity care. & $\begin{array}{l}\text { Denial or lack } \\
\text { of birth } \\
\text { companions: }\end{array}$ \\
\hline
\end{tabular}




\section{Figures}

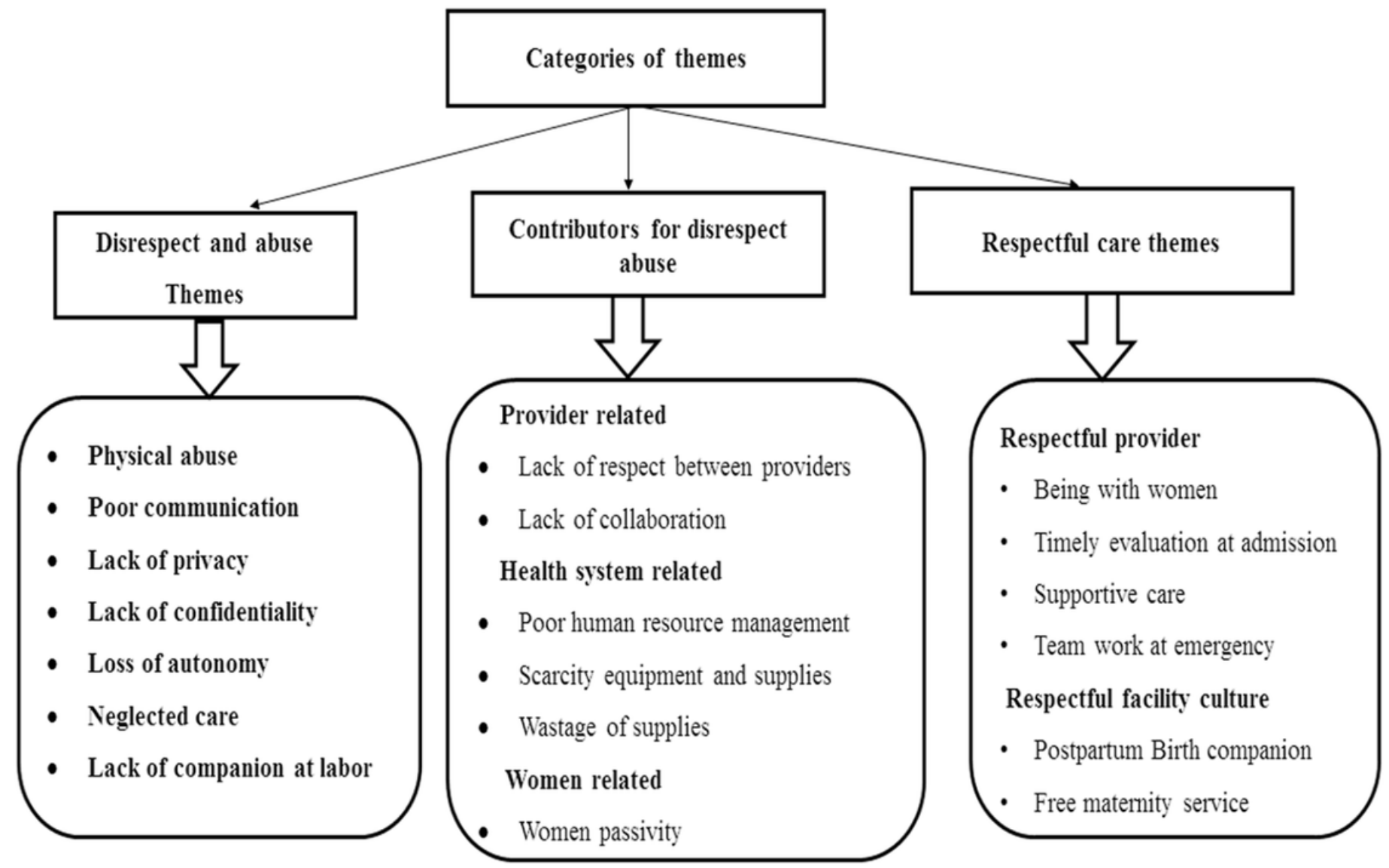

Figure 1

D\&A Themes, contributors for D\&A and Respectful Themes Identified from Participant Observation in a Tertiary Hospital. In South West Part of Ethiopia

\section{Supplementary Files}

This is a list of supplementary files associated with this preprint. Click to download.

- SupplementaryinformationMergarsaThoresenLulsegedLindahl.docx 